\title{
BOOLEAN ISOMORPHISM BETWEEN PARTIAL ORDERINGS OF CONVERGENT AND DIVERGENT SERIES AND INFINITE SUBSETS OF $\mathbb{N}$
}

\author{
PETER VOJTÁŠ
}

(Communicated by Andreas R. Blass)

\begin{abstract}
We prove that $\operatorname{RO}\left(\mathscr{P}(\mathbb{N}) / f\right.$ fin, $\left.\subseteq^{*}\right)$ is isomorphic under $\mathfrak{p}=$ $\operatorname{cf}\left(2^{\aleph_{0}}\right)$ to the Boolean completion of the comparison ordering of the absolutely divergent series (downwards) and under $\aleph_{1}=\operatorname{cf}\left(2^{\aleph_{0}}\right)$ to the Boolean completion of the ratio comparison ordering of absolutely convergent series (upwards).
\end{abstract}

\section{INTRODUCTION AND STATEMENT OF THE RESULTS}

One of the recent paradigms of set theory is the study of cardinal characteristics of the real line and structures connected with it (e.g., measure and category, the Čech-Stone remainder of $\mathbb{N}$, the Baire space $\mathcal{N}$, convergent series); see $[\mathrm{Ba}, \mathrm{vD}, \mathrm{F} 1, \mathrm{~F} 2, \mathrm{~J}, \mathrm{M}, \mathrm{Va}$ ]. The study of convergence went further along the lines of partial orderings of series that came from problems of real analysis; see, e.g., [Kh, V2]. While the comparison ordering of absolutely convergent series is upwards directed, our results show that the comparison ordering of absolutely divergent series (downwards), the ratiocomparison ordering of the absolutely convergent series (upwards), and almost inclusion on infinite subsets of natural numbers are (at least sometimes) similar. One of the ways to make this observation precise is to use the concept of the Boolean completion of a partially ordered set.

Recall, for a partial ordering $(P, \leqq)$, the topology generated by cuts of the form $\{x \in P: x \leq p\}, p \in P$ (i.e., the cut topology), produces the complete Boolean algebra of regular open subsets $\mathrm{RO}(P, \leqq)$ (see [Je]), which can be (for separative, but in a very natural sense also for nonseparative p.o.'s) considered as a Boolean completion of $P$.

Let $\ell^{\infty}$ denote the space of bounded infinite sequences of real numbers, $\ell^{1}$ the space of absolutely convergent series, and $\ell_{+}^{1}$ the set of absolutely convergent

Received by the editors January 8, 1991 and, in revised form, May 20, 1991.

1991 Mathematics Subject Classification. Primary 03E05, 40A05, 06E10, 03E35; Secondary 26A99, 28A99, 46B99.

Key words and phrases. (Ratio)comparison ordering of series; distributivity, base matrix, cardinal characteristics of Boolean algebras and of continuum.

This research was done while the author was a Research Fellow of the Alexander von HumboldtStiftung, Bonn at the Freie Universität Berlin. 
series taking nonzero real values. For $a, b \in \ell^{\infty} \backslash \ell^{1}$, define

$$
a \leq^{*} b \quad \text { iff } \quad(\exists n)(\forall k \geqq n)(|a(k)| \leqq|b(k)|)
$$

and for $a, b \in \ell_{+}^{1}$,

$$
a \unlhd^{*} b \quad \text { iff } \quad(\exists n)(\forall k \geqq n)\left(\left|\frac{a(k+1)}{a(k)}\right| \geqq\left|\frac{b(k+1)}{b(k)}\right|\right)
$$

(notice the orientation of inequalities $\unlhd^{*}$ and $\geqq$ ). We denote the corresponding Boolean completion of these (nonseparative) partial orders by $\operatorname{RO}\left(\ell^{\infty} \backslash \ell^{1}, \leq^{*}\right)$ and $\operatorname{RO}\left(\ell_{+}^{1}, \unlhd^{*}\right)$. Let $\mathscr{P}(\mathbb{N}) /$ fin be the set of infinite subsets of natural numbers, and for $A, B \in \mathscr{P}(\mathbb{N}) /$ fin, write

$$
A \subseteq^{*} B \text { iff }|A \backslash B|<\aleph_{0} .
$$

The Boolean completion of (the separative partial order) $\left(\mathscr{P}(\mathbb{N}) / f i n, \subseteq^{*}\right)$ is denoted by $\operatorname{RO}\left(\mathscr{P}(\mathbb{N}) /\right.$ fin, $\left.\subseteq^{*}\right)$.

Let

$$
\mathfrak{p}=\min \{|\mathscr{F}|: \mathscr{F} \subset \mathscr{P}(\mathbb{N}) / \text { fin is a filter base and } \bigwedge \mathscr{F}=\mathbf{0}\}
$$

(see $[\mathrm{Va}])$.

It is known that $\aleph_{1} \leqq \mathfrak{p} \leqq \operatorname{cf}\left(2^{\aleph_{0}}\right)$ and, e.g., under MA, $\mathfrak{p}=2^{\aleph_{0}}$ holds. In the second section we prove the following.

Theorem. (a) If $\mathfrak{p}=\operatorname{cf}\left(2^{\aleph_{0}}\right)$, then the Boolean algebras $\operatorname{RO}\left(\ell^{\infty} \backslash \ell^{1}, \leq^{*}\right)$ and $\operatorname{RO}\left(\mathscr{P}(\mathbb{N}) /\right.$ fin,$\left.\subseteq^{*}\right)$ are isomorphic. (b) If $\aleph_{1}=\operatorname{cf}\left(2^{\aleph_{0}}\right)$, then the Boolean algebras $\mathrm{RO}\left(\ell_{+}^{1}, \unlhd^{*}\right)$ and $\mathrm{RO}\left(\mathscr{P}(\mathbb{N}) /\right.$ fin,$\left.\subseteq^{*}\right)$ are isomorphic.

Problem. Is it provable in ZFC that $\operatorname{RO}\left(\ell^{\infty} \backslash \ell^{1}, \leq^{*}\right), \operatorname{RO}\left(\ell_{+}^{1}, \unlhd^{*}\right)$, and $\operatorname{RO}\left(\mathscr{P}(\mathbb{N}) /\right.$ fin,$\left.\subseteq^{*}\right)$ are isomorphic?

Our notation follows that of [Je] for p.o.'s and set theory, [BS] for Boolean algebras, [Va] for cardinal characteristics of the continuum, and [Fi] for real analysis.

\section{THE PROOF OF THE THEOREM}

The idea of the proof is to construct isomorphic dense trees in the algebras under consideration using the following.

Lemma 1 [BSV, BS]. Let $\tau, \lambda \geq \aleph_{0}, \kappa \geq 2$ be cardinals, $B a(\tau, \cdot, \kappa)$ nowhere-distributive Boolean algebra having a $\lambda$-closed dense subset $C$. Let $B$ be $(\rho, \cdot, 2)$-distributive for each $\rho<\tau$. If $\pi(B)=\kappa^{<\lambda}$, then there is a dense subset $T \subseteq C$ of $B$ such that $(T, \geq)$ is a tree of height $\tau$ and each $t \in T$ has $\kappa^{<\lambda}$ immediate successors.

As we do not know whether our orderings are homogeneous in the cardinal characteristics involved in Lemma 1, our arguments should be hereditary. Moreover, as our algebras are not separative and we do not know whether the cardinal characteristics are the same for the orderings in question and their (canonical) separative quotients, we have to be a little bit careful. Define " $x$ is compatible with $y$ in $P$ " (notation: $\left.\left.x\right|_{P} y\right)$ if $(\exists z \in P) \quad(z \leq x \& z \leq y)$; otherwise $x$ and $y$ are said to be incompatible, $x \perp_{P} y$. For $x \in P$, let 
$x^{-o}=\operatorname{int}(\operatorname{cl}(\{z \in P: z \leq x\}))$ be the interior of the closure of the cut generated by $x$ in the cut topology of $P$. The (canonical) equivalence

$$
x \approx_{P} y \text { iff }(\forall z)\left(\left.z\right|_{P} x \text { iff }\left.z\right|_{P} y\right) \text { iff } x^{-o}=y^{-o}
$$

and the induced ordering

$$
[x]_{\approx_{P}} \precsim_{P}[y]_{\approx_{P}} \text { iff } \quad(\forall z \leq x)\left(\left.z\right|_{P} y\right) \text { iff } \quad x^{-o} \subseteq y^{-o}
$$

makes $\left(P / \approx_{P}, \precsim_{P}\right)$ separative. Moreover,

$$
x \leq y \text { implies }[x]_{\approx_{P}} \precsim_{P}[y]_{\approx_{P}}
$$

and

$$
\left.x\right|_{P} y \text { iff }[x]_{\approx_{P} \mid P / \approx_{P}}[y]_{\approx_{P}} .
$$

The algebras $\operatorname{RO}(P, \leq)$ and $\operatorname{RO}\left(P / \approx_{P}, \precsim_{P}\right)$ are isomorphic. A separative partial ordering $(Q, \leq)$ can be embedded or w.l.o.g. can be considered as a dense subset of $\operatorname{RO}(Q, \leq)$.

For $a: \mathbb{N} \rightarrow \mathbb{R}$, we write $|a|(n)=|a(n)|, \mathscr{D}=\left\{|a|: a \in \ell^{\infty} \backslash \ell^{1}\right\}, \approx \mathscr{D}$ is the equivalence from (1) for $\mathscr{D}$ making $D=\mathscr{D} / \approx_{\mathscr{D}}$ separative with the induced ordering $\leq_{D}$ from (2). Write $B_{D}=\operatorname{RO}\left(D, \leq_{D}\right) \simeq \operatorname{RO}\left(\ell^{\infty} \backslash \ell^{1}, \leq^{*}\right)$. Similarly, write $\mathscr{K}=\left\{|a|: a \in \ell_{+}^{1}\right\}$ and $\approx_{\mathscr{K}}, K=\mathscr{K} / \approx_{\mathscr{K}}$ and $\leq_{K}, B_{K}=\mathrm{RO}\left(K, \leqq_{K}\right)$ $\simeq \operatorname{RO}\left(\ell_{+}^{1}, \unlhd^{*}\right)$. We denote the algebra $\operatorname{RO}\left(\mathscr{P}(\mathbb{N}) /\right.$ fin, $\left.\subseteq^{*}\right)$ by $B_{\mathbb{N}}$.

For $a \in \mathscr{K}$, let $f_{a}: \mathbb{N} \rightarrow \mathbb{R}^{+}$be defined as follows: $f_{a}(0)=a(0), f_{a}(n+1)=$ $\frac{a(n+1)}{a(n)}$; for $f: \mathbb{N} \rightarrow \mathbb{R}^{+}$define $a_{f}(n)=\prod_{i=0}^{n} f(i)$. We see that $a_{f_{a}}=a$ and that $\left(\ell_{+}^{1}, \unlhd^{*}\right)$ is isomorphic to

$$
\mathscr{F}=\left\{f_{a}: a \in \mathscr{K}\right\}=\left\{f: f: \mathbb{N} \rightarrow \mathbb{R}^{+} \text {and } \sum_{n=0}^{\infty} \prod_{i=0}^{n} f(i)<+\infty\right\}
$$

with the order $\geq^{*}$, where $\mathbb{R}^{+}$is the set of positive real numbers.

Observation 2. (A) For $a, b \in \mathscr{D}$,

$$
\left.a\right|_{\mathscr{D}} b \quad \text { iff }\left.[a]\right|_{D}[b] \text { iff } \sum_{n=0}^{\infty} \min (a(n), b(n))=+\infty
$$

and

(6) $[a] \leq_{D}[b]$ and $c \in[a]$ and $d \in[b]$ implies $[a]=[\min (c, d)]$.

(B) For $a, b \in \mathscr{K}$,

$$
\left.a\right|_{\mathscr{K}} b \quad \text { iff }\left.[a]\right|_{K}[b] \quad \text { iff } \quad \sum_{n=0}^{\infty} \prod_{i=0}^{n} \max \left(f_{a}(i), f_{b}(i)\right)<+\infty
$$

and

(8) $[a] \leq_{K}[b]$ and $c \in[a]$ and $d \in[b]$ implies $[a]=\left[a_{\max \left(f_{c}, f_{d}\right)}\right]$.

Proof. The proof is easy by (1), (2), (3), and (4); namely, (8) holds, thanks to the fact that in (2) we are allowed to restrict ourselves to $z \leq x$.

Lemma 3. The partial orderings $\left(K, \leqq_{K}\right)$ and $\left(D, \leqq_{D}\right)$ are atomless. Proof. Since the situation for $\left(D, \leqq_{D}\right)$ is quite transparent, we turn our attention to $K$. Given $a \in \mathscr{K}$, we construct $b, c \unlhd^{*} a$ such that $b$ and $c$ 
are incompatible in $\mathscr{K}$. Modifying an observation of Abel and Dini (see [ Fi, XI.375.4]), we prove that, for $\gamma(n)=\sum_{i=n}^{\infty} a(i)$, the series

$$
\begin{gathered}
b_{\sigma}(2 n+1)=\frac{a(2 n+1)}{\gamma^{1-\sigma}(2 n+1)}, \\
b_{\sigma}(2 n+2)=\frac{a(2 n+2)}{\gamma^{1-\sigma}(2 n+1)}, \quad b_{\sigma}(0)=a(0)
\end{gathered}
$$

and

$$
c_{\sigma}(2 n)=\frac{a(2 n)}{\gamma^{1-\sigma}(2 n)}, \quad c_{\sigma}(2 n+1)=\frac{a(2 n+1)}{\gamma^{1-\sigma}(2 n)}
$$

converge for $\sigma>0$. As

$$
\int \frac{1}{x^{1-\sigma}} d x=\frac{1}{\sigma} x^{\sigma}
$$

we have by the mean value theorem,

$$
\frac{1}{\sigma}\left(\gamma^{\sigma}(2 n+1)-\gamma^{\sigma}(2 n+3)\right)=(a(2 n+1)+a(2 n+2)) \frac{1}{\sigma} \frac{\partial x^{\sigma}}{\partial x}(\xi)
$$

for some $\xi$ such that $\gamma(2 n+3) \leq \xi \leq \gamma(2 n+1)$. But then

$$
\begin{aligned}
& \frac{1}{\sigma}\left(\gamma^{\sigma}(2 n+1)-\gamma^{\sigma}(2 n+3)\right)=\frac{a(2 n+1)+a(2 n+2)}{\xi^{1-\sigma}} \\
& \quad \geq \frac{a(2 n+1)+a(2 n+2)}{\gamma^{1-\sigma}(2 n+1)}=b_{\sigma}(2 n+1)+b_{\sigma}(2 n+2) .
\end{aligned}
$$

As $\sum_{n=0}^{\infty} \frac{1}{\sigma}\left(\gamma^{\sigma}(2 n+1)-\gamma^{\sigma}(2 n+3)\right)=\lim _{n \rightarrow \infty} \frac{1}{\sigma}\left(\gamma^{\sigma}(1)-\gamma^{\sigma}(2 n+3)\right)=\frac{1}{\sigma} \gamma^{\sigma}(1)$, we have that $b_{\sigma}$ converges (the case for $c_{\sigma}$ is similar). Now it suffices to show that $b=b_{\frac{1}{2}}$ and $c=c_{\frac{1}{2}}$ are incompatible in $\mathscr{K}$ and both are $\unlhd^{*}$ below $a$.

To this end, by (7) it suffices to show that $\max \left(f_{b}, f_{c}\right) \notin \mathscr{F}, f_{a} \leq^{*} f_{b}$, and $f_{a} \leq^{*} f_{c}$. Indeed,

$$
f_{b}(2 n+1)=\frac{a(2 n+1)}{a(2 n)} \sqrt{\frac{\gamma(2 n-1)}{\gamma(2 n+1)}}, \quad f_{b}(2 n+2)=\frac{a(2 n+2)}{a(2 n+1)}
$$

and

$$
f_{c}(2 n+1)=\frac{a(2 n+1)}{a(2 n)}, \quad f_{c}(2 n+2)=\frac{a(2 n+2)}{a(2 n+1)} \sqrt{\frac{\gamma(2 n)}{\gamma(2 n+2)}}
$$

Thus

$$
\max \left(f_{b}, f_{c}\right)(n+1)=\frac{a(n+1)}{a(n)} \sqrt{\frac{\gamma(n-1)}{\gamma(n+1)}}
$$

so, easily $f_{a} \leq^{*} f_{b}$, and $f_{a} \leq^{*} f_{c}$. Further, as

$$
\begin{aligned}
a_{\max \left(f_{b}, f_{c}\right)}(n) & =a(n) \sqrt{\frac{\gamma(0) \gamma(1) \cdots \gamma(n-2)}{\gamma(2) \gamma(3) \cdots \gamma(n)}} \\
& =\sqrt{\gamma(0) \gamma(1)} \frac{a(n)}{\sqrt{\gamma(n-1) \gamma(n)}} \geq \sqrt{\gamma(0) \gamma(1)} \frac{a(n)}{\gamma(n-1)},
\end{aligned}
$$


it is enough to show that $\sum_{n=0}^{\infty} \frac{a(n+1)}{\gamma(n)}=+\infty$. Suppose, on the contrary, that $\sum_{n=0}^{\infty} \frac{a(n+1)}{\gamma(n)}<+\infty$. Then tails converge to 0 , but

$$
\sum_{i=n}^{\infty} \frac{a(i+1)}{\gamma(i)} \geq \sum_{i=n}^{\infty} \frac{a(i+1)}{\gamma(n)}=\frac{\gamma(n+1)}{\gamma(n)} .
$$

So it suffices to have

$$
(\exists \alpha>0)(\forall k)(\exists n>k)\left(\frac{\gamma(n+1)}{\gamma(n)} \geq \alpha>0\right)
$$

for $a$ not to be an atom in $\mathscr{K}$. If not, then

$$
(\forall \alpha>0)\left(\exists k_{\alpha}\right)\left(\forall n>k_{\alpha}\right)(\gamma(n+1)<\alpha(a(n)+\gamma(n+1))),
$$

and hence $\frac{a(n+1)}{a(n)}<\frac{\alpha}{1-\alpha} ;$ that is,

$$
\lim _{n \rightarrow \infty} f_{a}(n)=0 \text {. }
$$

But define $b^{\prime}(2 n)=1 / 2^{n}, b^{\prime}(2 n+1)=1 / 2^{n}$ and $c^{\prime}(2 n+1)=1 / 2^{n}, c^{\prime}(2 n+2)$ $=1 / 2^{n}$. Both $b^{\prime}$ and $c^{\prime}$ are, because of (11), $\unlhd^{*}$-smaller than $a$ and incompatible in $\mathscr{K}$.

Lemma 4. The partial ordering $\left(K, \leqq_{K}\right)$ is $\sigma$-closed.

Proof. Take $\left\{a_{i}: i \in \mathbb{N}\right\} \subseteq \mathscr{K}$ such that $i<j$ implies $\left[a_{j}\right]<_{K}\left[a_{i}\right]$. Put $g_{i}(n)=\max \left\{f_{a_{j}}(n): j \leq i\right\}$. In $K$ we have that $\left[a_{g_{i}}\right]=\left[a_{i}\right]$. This can be proved by an induction of length $i$ using (8), and so $a_{g_{i}} \in \mathscr{K}$. So define by induction $n(0)=0$ and

$$
n(k+1)=\min \left\{j: j>n(k) \text { and } \sum_{i=j}^{\infty} a_{g_{k}}(i)<\frac{1}{2^{k}}\right\} .
$$

Define $g(i)=g_{k}(i)$ for $n(k) \leq i<n(k+1)$. As $a_{g} \unlhd^{*} a_{i}$ and $a_{g} \in \ell_{+}^{1}$, we are done. 
The following sets are dense in $P$ :

for $k \in \mathbb{N} \quad X_{k}=\{(s, a): k \in \operatorname{dom}(s)\}$ as $(s \cup a\lceil[(k+1) \backslash \operatorname{dom}(s)], a)=$ $s_{a, k} \leq(s, a)$

for $m \in \mathbb{N}, Y_{m}=\left\{(s, a): \sum_{n \in \operatorname{dom}(s)} s(n)>m\right\}$ as $s_{a, k}$ for sufficiently large $k$ lies in $Y_{m}$;

for $\alpha<\kappa \quad Z_{\alpha}=\left\{(s, a): a \leq a_{\alpha}\right\}$ as $\left(s, \min \left(a, a_{\alpha}\right)\right) \in P$.

Let $G \subseteq P$ be a filter that meets every $X_{k}, Y_{m}$, and $Z_{\alpha}$ for $(k, m, \alpha) \in$ $\mathbb{N} \times \mathbb{N} \times \kappa$. Put

$$
f=\bigcup\{s:(s, a) \in G\} .
$$

Then $f \in \mathscr{D}$ and $f \leq^{*} a_{\alpha}$ for every $\alpha<\kappa$. To see the latter, notice that if $(s, a) \in Z_{\alpha} \cap G$ is fixed and $(r, b) \in G \cap X_{k}$ for $k>\operatorname{dom}(s)$ is arbitrary, then as $G$ is centered, $s \subseteq r$, and

$$
(\forall n \in \operatorname{dom}(r) \backslash \operatorname{dom}(s))\left(r(n) \leq \min (a(n), b(n)) \leq a_{\alpha}(n)\right),
$$

i.e., $(\forall k>\operatorname{dom}(s))\left(f(k) \leq a_{\alpha}(k)\right)$.

Corollary 6. hsat $\left(K, \leqq_{K}\right)=\operatorname{hsat}\left(D, \leqq_{D}\right)=2^{\aleph_{0}}$.

Proof. Using atomlessness and $\sigma$-closedness, construct a splitting binary tree of length $\aleph_{0}$ consisting of elements of $K$ (in $D$ similarly). As there are $2^{\aleph_{0}}$-many branches and below each of them there is a nonzero element, we are done.

Corollary 7. The algebra $\operatorname{RO}\left(D, \leqq_{D}\right)$ is $\rho$-distributive for every $\rho<\mathfrak{p}$, and the algebra $\mathrm{RO}\left(K, \leqq_{K}\right)$ is $\aleph_{0}$-distributive.

Proof. As closedness of a dense subset implies distributivity, the proof is easy.

Lemma 8. Both $\mathrm{RO}\left(K, \leqq_{K}\right)$ and $\mathrm{RO}\left(D, \leqq_{D}\right)$ are not $\operatorname{cf}\left(2^{\aleph_{0}}\right)$-distributive.

Proof. Take $K=\bigcup K_{\alpha}, \alpha<\operatorname{cf}\left(2^{\aleph_{0}}\right)$ such that $\left|K_{\alpha}\right|^{+}<2^{\aleph_{0}}$. Then as $\left|K_{\alpha}\right|^{+}<$ hsat $(K)$ by the refining lemma of [BV], there is a disjoint refinement $P_{\alpha}$ of $K_{\alpha}$. The matrix $\left\{P_{\alpha}: \alpha<\operatorname{cf}\left(2^{\aleph_{0}}\right)\right\}$ cannot have a common refinement as $K$ is not atomary. (The case of $D$ is the same.)

Proof of the theorem. Note that all our observations are valid hereditarily.

(a) Under $\mathfrak{p}=\operatorname{cf}\left(2^{\aleph_{0}}\right)$, the algebra is homogeneous in the cardinal characteristics involved in Lemma 1 , and the conditions of Lemma 1 are fulfilled with $\tau=\operatorname{cf}\left(2^{\aleph_{0}}\right), \kappa=2$, and $\lambda=\mathfrak{p}$, the algebra $B_{D}$ is $(\mathfrak{p}, \cdot, 2)$-nowheredistributive and has a $\mathfrak{p}$-closed dense set (namely, $D)$, is $(\rho, \cdot, 2)$-distributive for every $\rho<\mathfrak{p}$, and $\pi\left(B_{D}\right)=2^{\aleph_{0}}=2^{<\mathfrak{p}}$. Take $T_{D}=\left\{P_{\alpha}: \alpha<\mathfrak{p}\right\} \subseteq D$ from the assertion of Lemma 1, i.e., $T_{D}$ is a tree of height $\mathfrak{p}$ such that each $t \in T_{D}$ has $2^{\aleph_{0}}$ immediate succesors. Notice, moreover, that all maximal branches are of length $\mathfrak{p}$, i.e., all chains are long in the terminology of [BPS, D]. Then, notice that $\bigcup\left\{P_{\alpha+1}: \alpha<\mathfrak{p}\right\}$ is isomorphic to $\bigcup_{\alpha<p}{ }^{\alpha}\left(2^{\aleph_{0}}\right)$ ordered by the inverse inclusion (the canonical dense set of the complete Boolean algebra $\operatorname{Col}\left(2^{\aleph_{0}}, \mathfrak{p}\right)$ ). Moreover, using results of [BPS], under $\mathfrak{p}=\operatorname{cf}\left(2^{\aleph_{0}}\right)$ the same is the case for $B_{\mathrm{N}}$.

(b) Under $\aleph_{1}=\operatorname{cf}\left(2^{\aleph_{0}}\right)$, the conditions of Lemma 1 are fulfilled for $B_{K}$ with $\lambda=\tau=\aleph_{1}, \kappa=2$, and $K$ is a $\sigma$-closed dense subset of $B_{K}$.

\section{CONCLUDING REMARKS}

3.1. Observe that some of the cardinal characteristics from [Va] can be defined for $\mathrm{RO}\left(K, \leqq_{K}\right)$ and $\mathrm{RO}\left(D, \leqq_{D}\right)$. Consider $\mathfrak{t}_{K}, \mathfrak{h}_{K}, \mathfrak{t}_{D}, \mathfrak{h}_{D}$. We proved in 
fact that if

$$
\mathfrak{t}_{D}=\mathfrak{h}_{D}=\mathfrak{t}=\mathfrak{h}
$$

then

$$
\operatorname{RO}\left(\ell^{\infty} \backslash \ell^{1}, \leq^{*}\right) \simeq \operatorname{RO}\left(\mathscr{P}(\mathbb{N}) / \text { fin }, \subseteq^{*}\right)
$$

We show that this assumption is not necessary.

Example. It is consistent with ZFC that $\mathfrak{t}<\mathfrak{h}$ and $B_{D} \simeq B_{\mathbb{N}}$.

Construction. Our construction uses the model from Theorem 2.1 of [D]. Start with a model of $\mathrm{GCH}$ and force with a c.c.c. partial order $Q$ such that in $V^{Q}$ we have $M A$ and $2^{\aleph_{0}}=\aleph_{2}$, and hence $B_{D} \simeq B_{\mathbb{N}}$. After this force with $P=\left(\bigcup_{\alpha<\aleph_{1}}{ }^{\alpha} 2\right) \cap V, Q * P$ is in fact the product $Q \times P . P$ adds no new countable subsets of $V^{Q}$, and hence $D$ and $\mathscr{P}(\mathbb{N}) /$ fin in $V^{Q}$ and $V Q \times P=W$ are the same, and so they still have isomorphic dense subsets, and hence, in $W$, $B_{D} \simeq B_{\mathbb{N}}$. As proved by Dordal, in $W$ we have $\mathfrak{t}=\aleph_{1}$ and $\mathfrak{h}=\aleph_{2}$.

3.2. We would like to emphasize the problem from the introduction by the following: the problem of provability in ZFC of $B_{D} \simeq B_{K} \simeq B_{\mathbb{N}}$ is a kind of question whether the 2-valued structure $(\mathscr{P}(\mathbb{N}) /$ fin) and real-valued structures $(\mathscr{D}, \mathscr{K})$ are similar. Let us recall from [V1] a problem of a similar kind (see also [Va]). Let

$$
\mathfrak{r}=\min \left\{|\mathscr{R}|: \mathscr{R} \subseteq \mathscr{P}(\mathbb{N}) / \text { fin and }(\forall f: \mathbb{N} \rightarrow\{0,1\})(\exists R \in \mathscr{R}) \lim _{n \in R} f(n) \text { exists }\right\}
$$

and

$$
\mathfrak{r}_{\sigma}=\min \left\{|\mathscr{R}|: \mathscr{R} \subseteq \mathscr{P}(\mathbb{N}) / \text { fin and }\left(\forall f \in \ell^{\infty}\right)(\exists R \in \mathscr{R}) \lim _{n \in R} f(n) \text { exists }\right\} .
$$

Is $\mathfrak{r}=\mathfrak{r}_{\sigma}$ provable in ZFC? Easily $\mathfrak{r} \leq \mathfrak{r}_{\sigma}$, and Just [Ju] pointed out that if $\mathfrak{r}<\mathfrak{r}_{\sigma}$, then either $\mathfrak{r}<\mathfrak{u}$ or $\mathfrak{u} \geq \aleph_{\aleph_{0}+1}$, and there is a measurable cardinal in an inner model. Is our problem $B_{D} \simeq B_{K} \simeq B_{\mathbb{N}}$ of the same kind, i.e., are large cardinals involved? Note that the structure of $\left(\ell^{1}, \leq^{*}\right)$ depends on the set theory but large cardinals are up to now not involved.

3.3. Hierarchies of classical tests were constructed using a chain of parameters in $\left(\ell_{+}^{1}, \unlhd^{*}\right)$ consisting of monotone series (e.g., Raabe's, Bertrand's ratiocomparison tests, ...). We do not even know whether, for

$$
\mathscr{F}_{1}=\left\{f \in \mathscr{F}: \forall_{n}^{\infty} f(n) \leq 1\right\}
$$

$\operatorname{RO}\left(\mathscr{F}_{1}, \geq^{*}\right)$ is atomless. If this is the case, then it is also isomorphic to $B_{\mathbb{N}}$ at least under $\aleph_{1}=\operatorname{cf}\left(2^{\aleph_{0}}\right)$.

\section{REFERENCES}

[BPS] B. Balcar, P. Pelant, and P. Simon, The space of ultrafilters on $\mathbb{N}$ covered by nowhere dense sets, Fund. Math. 90 (1980), 11-24.

[BS] B. Balcar and P. Simon, Disjoint refinement, Handbook of Boolean algebras (J. D. Monk and R. Bonnet. eds.), North-Holland, Amsterdam, 1989, pp. 333-388.

[BSV] B. Balcar, P. Simon, and P. Vojtáš, Refinement properties and extensions of filters in Boolean algebras, Trans. Amer. Math. Soc. 267 (1981), 265-283 . 
[BV] B. Balcar and P. Vojtáš, Refining systems on Boolean algebras, Set Theory and Hierarchy Theory V (A. Lachlan, M. Srebrny, and A. Zarach, eds.), Lecture Notes in Math., vol. 619, Springer-Verlag, Berlin, 1977, pp. 45-58.

[Ba] T. Bartoszynski, Additivity of measure implies additivity of category, Trans. Amer. Math. Soc. 281 (1984), 209-213.

[Be] M. G. Bell, On the combinatorial principle $P(\mathfrak{c})$, Fund. Math. 114 (1981), 149-157.

[D] P. L. Dordal, Independence results concerning some combinatorial properties of continuum, Ph.D. Thesis, Harvard Univ., Cambridge, MA, 1982.

[vD] E. K. van Douwen, The integers and topology, Handbook of Set-Theoretic Topology (K. Kunen and J. E. Vaughan, eds.), North-Holland, Amsterdam, 1984, pp. 111-167.

[Fi] G. M. Fichtenholz, The course of diferential and integral calculus, Fizmatgiz, Moscow, 1959.

[F1] D. H. Fremlin, Cichoń's diagram, Séminaire Initiation á l'Analyse , 23e année, G. Choquet, M. Rogalski, J. Saint-Raymond, 1984.

[F2] _ Consequences of Martin's axiom, Cambridge Univ. Press, Cambridge, MA, 1984.

[Je] T. Jech, Set theory, Academic Press, New York, 1978.

[J] H. Judah, Set theory of reals: measure and category, preprint, 1990.

[Ju] W. Just, A letter to the author, 31.10.1989.

[Kh] N. N. Kholshchevnikova, Unsolvability of several questions of convergence of series and sequences, Mat. Z. 34 (1983), 711-718. (Russian)

[M] A. W. Miller, Some properties of measure and category, Trans. Amer. Math. Soc. 266 (1981), 93-144.

[Va] J. E. Vaughan, Small uncountable cardinals in topology, Open Problems in Topology (J. van Mill and G. M. Reed, eds.), North-Holland, Amsterdam, 1990, pp. 195-218.

[V1] P. Vojtáš, Cardinalities of possibly noncentered systems of subsets of $\mathbb{N}$ which reflect some qualities of ultrafilters, p-points and rapid filters, Proc. Internat. Conf. on Topology and its Applications (Baku, 1987) (submitted).

[V2] - Set theoretic characteristics of summability of sequences by regular (Toeplitz) matrices, Comment. Math. Univ. Carolin. 28 (1987), 173-183.

Mathematical Institute, Slovak Academy of Sciences, Jesenná 5, 04154 Košice, CzechOSLOVAKIA

E-mail address: vojtas@csearn.bitnet and vojtas@upjs.cs 